Pak. j. sci. ind. res. Ser. B: biol. sci. 2020 63B(3) 169-178

\title{
Isolation, Cloning and Characterization of a Constitutive Plant from Potato Aquaporin Gene
}

\author{
Hira Mubeen $^{\text {ab*, Rubab Zahra Naqvi }}{ }^{\mathrm{b}}$, Ammara Masood ${ }^{\mathrm{ab}}$, Mushtaq A. Saleem ${ }^{\mathrm{a}}$, \\ Aftab Bashirc and Shahid Razad \\ ${ }^{a}$ Department of Biotechnology, University of Central Punjab Lahore, Pakistan \\ ${ }^{b}$ National Institute for Biotechnology and Genetic Engineering, Faisalabad \\ ${ }^{\circ}$ Lahore Garrison University, Lahore, Pakistan \\ ${ }^{\mathrm{d}}$ Forman Christian College University, Lahore, Pakistan
}

(received March 16, 2017; revised October 29, 2018; accepted January 16, 2019)

\begin{abstract}
Plasma membrane intrinsic proteins (PIP1) are the most common integral membrane proteins belong to a larger family of intrinsic aquaporin proteins. They are member of aquaporin gene family and have gained importance as highly expressed genes in plants. In this study, the promoter of aquaporin PIP1 gene was identified, analyzed and retrieved from high throughput genomic sequence (HTGS) database. The cis-acting regulatory elements, transcription start sites and transcription factor binding sites of selected promoter were identified through different bio-informatics tools. Many light responsive, phytohormone, stress and defense related cis-regulatory elements were detected in PIP1 promoter region indicating its role as a constitutive promoter. The PIP1 promoter was isolated from Solanum tuberosum. It was initially cloned in TA vector (pTZ57R/T) and later transferred to plant expression binary vectors, pGR1 and pGA482 for transient and stable expression studies in tobacco. The GUS expression results of PIP1 promoter in different tobacco tissues showed its functional importance in regulating gene expression in a constitutive manner. Further, it was concluded that the PIP1 aquaporin promoter is constitutively expressed with a strength equivalent to CaMV $2 \times 35 \mathrm{~S}$ promoter. These findings indicated the significance of isolated promoter for genetic engineering of plants for crop improvement.
\end{abstract}

Keywords: HTGS, aquaporin, GUS, vector, promoter

\section{Introduction}

Plant aquaporins are located intracellularly with diversity of isoforms. Genome sequencing has showed different number of aquaporin genes with known functions. Studies showed presence of nearly 35 genes in Arabidopsis genome, 33 in rice and 36 in maize (Sakurai et al., 2005; Chaumont et al., 2001; Johanson et al., 2001; Quigley et al., 2001). The genome sequencing of Populus trichocarpa has showed nearly 55 full-length aquaporins. However, the study of different plants also indicated the presence of aquaporin isoforms in Triticum aestivum, Nicotiana tabacum and other plants. Plant aquaporins have been divided into different sub families based on sequence similarity. Some of the common families include the plasma membrane intrinsic proteins (PIPs), nodulin26-like intrinsic proteins (NIPs), tonoplast intrinsic proteins (TIPs) and some of the basic intrinsic proteins (SIPs) (Johanson and Gustavsson, 2002; Chaumont et al., 2001; Johanson et al., 2001).

Aquaporins of the plasma membrane (PIP's) family are recognized as the highly conserved subfamily in plants.

*Author for correspondence; E-mail: hira_sh@hotmail.com
Members of the plasma membrane intrinsic protein are important for the maintenance of the plant water status through the control of cell and tissue hydraulics. PIPs are divided into two of the phylogenetic groups, PIP1 and PIP2 which are important for different water channel activities. However, PIP1 isoforms are conserved and share more than $90 \%$ amino acid sequence identity. They have functional importance in phloem loading, transport and unloading (Bots et al., 2005; Lopez et al., 2003; Martre et al., 2002).

Promoters are key regulators of plant gene expression and regulation. To improve the quality of crops, suitable promoters are necessarily required (Naqvi et al., 2017). Isolation and characterization of useful plant promoters is routinely required for genetic manipulation of plants and is important in achieving controlled gene expression in transgenic plant development programs. The PIP1 aquaporin genes are abundantly expressed in dicot plants. Therefore, PIP1 gene was selected to characterize as a strong constitutive promoter of plant origin. This study was performed to identify, evaluate and characterize the promoter sequence of PIP1 gene for 
its transient and stable expression studies in Nicotiana tabacum via Histochemical GUS assay.

\section{Materials and Methods}

Bioinformatics approach for evaluation of the promoter. The query sequence of PIP1 gene was extracted and assessed by using various bioinformatics approaches. The Uniprot (www.expasy.ch) was used to attain the nucleotide sequence of PIP1, based on its high expression level in dicots. Further, to isolate the promoter region, the PIP1 gene sequence was explored in BLAST against High throughput Genomic Sequences (HTGS) database for similarity searches. Results showed HTGS matches reported for Solanum tuberosum. The start codon of gene was searched through HTGS. Nearly, about $3 \mathrm{~Kb}$ upstream region of PIP1 gene was selected and analyzed for identification of transcription initiation site (TSS) using the software, BDGP-Neural Network Promoter Prediction. The BLAST results for regulatory sequence were useful for verification against all previously patent sequences.

Analysis of cis-regulatory elements in promoter region. The cis-regulatory elements of PIP1 gene promoter was screened using PlantCARE software. For analysis of certain factors which act as a key regulator for gene expression regulation, the Plant PAN software was used.

Isolation of selected promoter sequence. To isolate DNA from fresh leaves of $S$. tuberosum (potato), CTAB method was used (Rogers and Bendich, 1985). The isolated DNA was used for amplification of PIP1 promoter by using conventional PCR. To select a fulllength promoter sequence, four different sets of primer pairs were designed on PIP1 promoter sequence. These primer sequences are given below:

Primer pair-1: AqpSoTubF-3 5'

GATGAGCTCTTAGTATTTTCCTT

CGGCTTA 3'

AqpSoTubR-1 5'

GCCAAGCTTAATTCTTCAAGAT

TATGCCAAAG 3'

Primer pair-2: AqpSoTubF-4 5'

GATGAGCTCTTAGTATTTTCCTT

CGGCTTAA 3'

AqpSoTubR-2 5'

GACAAGCTTTTTTACAAAAAAA

TTCTTCAAG 3'

Primer pair-3: AqpSoTubF-5 5'

GATGAGCTCTTAGTATTTTCCTT
CGGCTTAAT 3'

AqpSoTubR-4 5'

GACAAGCTTTTTTACAAAAAAA

TTCTTCAAGATTATGC 3'

Primer pair-4: AqpSoTubF-6 5'

GATGAGCTCCCTTCGGCTTAAT

TTATATGAC 3'

AqpSoTubR-3 5'

GACAAGCTTTTTTACAAAAAAA

TTCTTCAAGATTATG

Conferring to the particular cloning approach, two restriction sites were added to forward primer, one for the SacI (GAGCTC) and other to reverse primer for HindIII site (AAGCTT). For optimization of best primer pair with suitable annealing temperature, the gradient PCR was used. The primer pair AqpSoTubF-6 (5' ATGAGCTCCCTTCGGCTTAATTTATATGAC $3^{\prime}$ ) and AqpSoTubR-3 (5' GACAAGCTTTTTTACAA AAAAATTCTTCAAGATTATG) was finally used to amplify the promoter region. The PCR single reaction was set as: $4 \mu \mathrm{L} \mathrm{MgCl}_{2}(25 \mathrm{mM}), 4 \mu \mathrm{L}$ Taq Polymerase buffer (10X), $1 \mu \mathrm{L}$ dNTP (10 mM), $1 \mu \mathrm{L}$ of forward and reverse primer $(50 \mathrm{ng} / \mu \mathrm{L}), 1 \mu \mathrm{L}$ Taq DNA polymerase ( 2.5 unit $/ \mu \mathrm{L}), 5 \mu \mathrm{L}$ DNA (1:40 dilution), $35 \mu \mathrm{L}$ $\mathrm{H}_{2} \mathrm{O}$ under the conditions of initial denaturation at 92 ${ }^{\circ} \mathrm{C}$ for $4 \mathrm{~min}$, followed by 40 repeated cycles of denaturation at $94{ }^{\circ} \mathrm{C}$ for $1 \mathrm{~min}$, annealing at $46^{\circ} \mathrm{C}$ for $1 \mathrm{~min}$, extension at $72{ }^{\circ} \mathrm{C}$ for $1 \mathrm{~min}{ }^{\circ} \mathrm{C}$ for $1 \mathrm{~min}$ and a final extension at $72{ }^{\circ} \mathrm{C}$ for $5-10 \mathrm{~min}$.

Cloning of PIP1 promoter in TA (pTZ57R/T) vector. The amplified products of promoter sequence was ligated in a general purpose cloning vector TA (pTZ57R/T) followed by transformation in TOP10 cells. The cloned fragment of aquaporin PIP1 promoter was named TA-Aqu, which was further confirmed through restriction digestion, sequencing and PCR analysis.

Cloning of PIP1 promoter in expression vector. To study the expression of reporter gene, the subject promoter sequence was transferred to another vector (pGR1), which is a transient expression vector and modified form of pJITT166 consisting of GUS with intron. The pGR1 plasmid contains GUS with intron under $2 \times 35 \mathrm{~S}$ promoter and occupies a size of $5.8 \mathrm{~kb}$ followed by CaMV terminator. The $2 \times 35 \mathrm{~S}$ promoter in pGR1 was replaced by PIP1 promoter after digestion of pGR1 with SacI and HindIII. The cloned PIP1 gene in pGR1 was named pGRAquP and was verified through restriction analysis, PCR and sequencing. 
Transient expression of GUS under PIP1 promoter. To evaluate the activity of PIP1 promoter, the transient expression studies were carried out in the model plant Nicotiana tabacum (tobacco) by using reporter gene (GUS). The selected vector for PIP1 promoter, pGRAqup was bombarded by using Biolistic particle delivery system available at lab (PDS1000 He). The ethanol treatment was carried out for stopping screen, rupture discs and macrocarriers to fulfill all precautionary measures for successful results. The two pGR1 vectors were selected for bombardment, one under control of CaMV 35S promoter and second under the control of PIP1 promoter. The samples of fresh tobacco leaves and stems were selected from green house, sterilized with water and allowed to dry for few minutes. Afterword's, the samples were placed at the center of petri plate containing MS medium and construct was coated with gold particles (Battraw and Hall, 1990; Murashige and Skoog, 1962). The selected tissues were exposed for bombarded using 1100 psi rupture disks with a $5 \mathrm{~cm}$ target distance at 29 inch $\mathrm{Hg}$ vacuum. Finally, samples were wrapped in a dish and incubated at $30{ }^{\circ} \mathrm{C}$ for $24 \mathrm{~h}$.

Analysis of ß-glucuronidase expression. Histochemical localization of GUS enzyme activity was carried out using 5-bromo-4-chloro-3-indolyl-b-D-glucuronide (XGlc). All types of tissues were covered with X-gluc staining solution, and vacum infiltrated. The samples were incubated at $37{ }^{\circ} \mathrm{C}$ in dark for overnight. At the completion of incubation time, tissues were treated with different dilutions of ethanol to remove the pigmentation due to chlorophyll. The localized expression of GUS in all the tissues was intermittently monitored. The detailed observation was carried out with light microscope. Histochemically stained tissues i.e. control and positive transformants were photographed using a microscope attached to a digital microscope camera.

Cloning of PIP1 in plant transformation vector (pGA482). The complete expression cassette of PIP1 promoter in pGR1 vector was digested with XhoI resulting in sticky ends. The end filling technique was further used to convert sticky ends into blunt ends. The reaction was stopped after $10 \mathrm{~min}$ and isolated plasmid DNA was phenol treated, ethanol washed and resuspended in $20 \mu \mathrm{L}$ of $\mathrm{H}_{2} \mathrm{O}$. Moreover, the second digestion was performed by digesting with SacI to release the expression cassette from the parent vector. The mixture was again treated with phenol and precipitated in a similar way. However, the digested cassette was cloned in plant expression vector pGA482 and transformed in E. coli (TOP10) cells. The cloned PIP1 promoter cassette in pGA482 was named as pGAAqup. The clones were further confirmed and verified by using restriction and PCR. Finally, the plant expression construct was transformed in Agrobacterium strain LBA4404 by electroporation for Agrobacterium-mediated transformation in tobacco plants.

Agrobacterium mediated transformation in tobacco. Leaf disk co-cultivation. pGA Aqup transformed in LBA4404 was cultured in LB broth containing antibiotics with relative concentrations required for Agrobacterium growth. Furthermore, tobacco seeds were germinated and grown in vitro on MS medium (Murashige and Skoog, 1962). From freshly grown plants of tobacco, leaves were taken and cut into about 30-40 leaf disks. These leaf disks were exposed to the grown agrobacterium culture of pGAAqup at room temperature for about half an hour. Inoculated leaf disks were transferred to the Whatman filter papers and then blotted thoroughly.

\section{Selection and regeneration of transformed callus.} The leaf disks co-cultivated with AgrobacteriumpGAAqup were collected, washed 4-5 times with MS liquid medium containing Cefotaxime $(250 \mu \mathrm{g} / \mathrm{mL})$ to remove extra growth of Agrobacterium and blotted to remove excessive water. These leaf discs were then transferred to callus induction were transferred to cocultivation MS medium (supplemented with 1-naphthaleneacetic acid, $0.1 \mathrm{mg} / \mathrm{L}$ and 6-benzylaminopurine $1 \mathrm{mg} / \mathrm{L}$ ) and incubated at $26^{\circ} \mathrm{C}$ for 2 days with no light. This was followed by transferring discs to selection medium for 3-4 weeks. At the development of 7-10 leaves, the putative transgenic plants were transferred to rooting media sealed with PVC cling film and incubated in growth chamber. Plants were carefully transferred to the pots containing mixture of loamy soil and sand (1:1), covered with water soaked polythene bags and placed at $25{ }^{\circ} \mathrm{C}$ in the green house. Plants were lightly irrigated after every 2 days interval. After 7-10 days, the plants well adapted to soil were gradually exposed to green house environment by cutting the polythene coverings from upper side. The putative transgenic and control tobacco plants were routinely observed and noted for their morphological appearance during developmental stages.

Molecular analysis of putative transgenic plants. Young leaves from putative transgenic plants of 
pGAAqup and negative control tobacco plant were selected for DNA isolation by CTAB method. The Presence of PIP promoter in transgenic tobacco plants was performed by PCR of plant genomic DNA by using promoter specific primer pair 4 (AqpSoTubF-6 and AqpSoTubR-3).

\section{Results and Discussion}

Detection and retrieval of the promoter sequences. The PIP1 gene sequence was taken from Genbank at NCBI and was searched in BLAST against HTGS which got a hit in HTGS for $S$. tuberosum (accession number AC233511). About $3 \mathrm{~kb}$ of the nucleotide sequence upstream to PIP1 gene was selected. The evaluation of promoter sequences was performed using different bioinformatics tools. Based on analysis of regulatory regions having transcription start site and cis-regulatory elements, a $1770 \mathrm{bp}$ promoter region of gene was selected. The regulatory sequence when BLAST searched against the patent sequences, it was verified that it is not regulated by any filed patent.

Detection of transcription start site (TSS) in promoter sequence. Transcription start site is a sequence in a promoter that functions as the RNA polymerase binding site. Its identification assures that the sequence is truly a promoter region. The BDGP promoter analysis revealed following sequence with a probability $90 \%$ for PIP1 promoter.

\section{GAATTCCCTCTATAAAAAGAATCACCATCTCC ACAATCTTGACAACACAC}

The capital $G$ in the sequence represents the predicted transcription start site. The transcription start site for PIP1 promoter was detected at nucleotide position 1710 of the $1770 \mathrm{bp}$ promoter.

Cis-regulatory elements associated with the promoter. PlantCARE software analysis revealed several types of motifs dispersed over the entire promoter. The most frequent motif was observed to be light responsive element, while stress, infection and hormone response motifs were also found scattered throughout the promoter region. The predicted regulatory elements for PIP1 promoter are given in Table 1.

Identification of transcription factor binding site through plant PAN. Plant PAN software (www. plantpan.mbc.nctu.edu.tw/) identifies the transcription factors that are key regulators of gene expression. The putative transcription factor binding sites (TFBs) of PIP1 gene promoter are illustrated in Table 1.
Isolation of promoter from respective plant specie. After optimization of annealing temperature and primer pairs, best primer pair and annealing temperature was selected to get the appropriate fragment size of the promoter. The selected primer pair successfully amplified a $1.7 \mathrm{~kb}$ PIP1 promoter fragment from genomic DNA of $S$. tuberosum. The best annealing temperature for this primer pair was observed to be $48^{\circ} \mathrm{C}$.

Cloning of promoter in TA vector. PCR product of was ligated in TA $(\mathrm{pTZ} 57 \mathrm{R} / \mathrm{T})$ vector and after transformation the clones of PIP1 promoter was confirmed in TA (pTZ57R/T) vector. TA Clones of PIP1 promoter (TA-Aqu) was confirmed via restriction and PCR (Fig. 1 a \& b). Clones were also confirmed by DNA sequencing on an ABI3100 DNA sequencer using BigDye Terminator sequencing kit version 3.1.

Cloning of PIP1 promoter in a derivative of pJIT166 (pGR1). The pGR1 was isolated and restriction analysis was performed. The $2 \times 35 \mathrm{~S}$ promoter is $750 \mathrm{bp}$ and digestion of pGR1 with SacI and Hind III released the 2x35S promoter (Fig. 2a). PIP1 promoter was amplified with the promoter specific primers using its clone in TA (TA-Aqu) as template. The amplified product was digested with SacI and Hind III to get sticky ends. The $1770 \mathrm{bp}$ fragment representing PIP1 promoter was then used for ligation into the respective sites in the transient expression vector and transformed in E.coli (TOP10) cells. Clones PIP1 aquaporin promoter
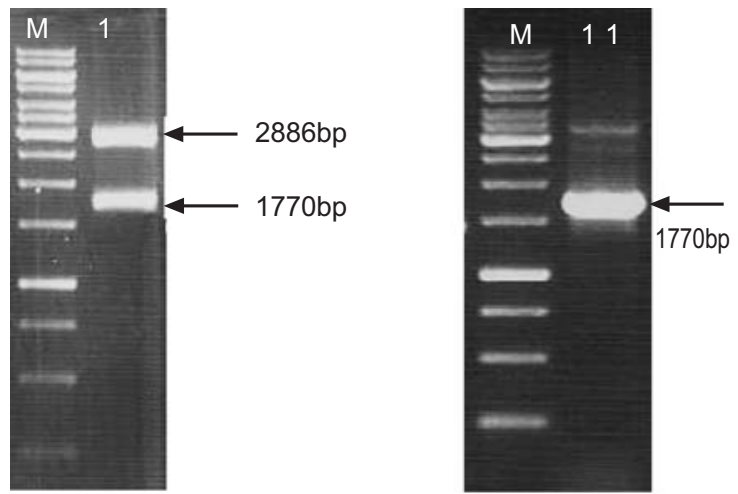

Fig. 1. Verification of promoter clones in TA (pTZ57R/T). (a) confirmation by restriction analysis. $\mathrm{M}: 1 \mathrm{~Kb}$ DNA ladder, lane 1: digestion of PIP1 aquaporin promoter clone with SacI and Hind III indicating a $1.7 \mathrm{~kb}$ insert; (b) confirmation by PCR. M: $1 \mathrm{~Kb}$ DNA ladder, lane 1: PCR amplification of PIP1 promoter. 
Table 1. Cis-regulatory motifs in PIP1 aquaporin promoter

\begin{tabular}{|c|c|c|c|c|}
\hline Site name & Organism & Position & Sequence & Function \\
\hline AE-box & Arabidopsis thaliana & 527 & AGAAACAA & part of a module for light response \\
\hline AE-box & Arabidopsis thaliana & 557 & AGAAACAT & part of a module for light response \\
\hline ATCT-motif & Arabidopsis thaliana & 1000 & AATCTAATCT & part of a conserved DNA module involved in light responsiveness \\
\hline AT-rich element & Glycine $\max$ & 1209 & ATAGAAATCAA & binding site of AT-rich DNA binding protein (ATBP-1) \\
\hline AuxRR-core & Nicotiana tabacum & 646 & GGTCCAT & cis-acting regulatory element involved in auxin responsiveness \\
\hline Box 4 & Petroselinum crispum & 379 & ATTAAT & part of a conserved DNA module involved in light responsiveness \\
\hline Box III & Pisum sativum & 761 & CATTTACACT & protein binding site \\
\hline Box-W1 & Petroselinum crispum & 510 & TTGACC & fungal elicitor responsive element \\
\hline CAAT-box & Brassica rapa & 1365 & CAAAT & common cis-acting element in promoter and enhancer regions \\
\hline CAAT-box & Brassica rapa & 1385 & CAAAT & common cis-acting element in promoter and enhancer regions \\
\hline GA-motif & Arabidopsis thaliana & 940 & ATAGATAA & part of a light responsive element \\
\hline GA-motif & Arabidopsis thaliana & 1105 & ATAGATAA & part of a light responsive element \\
\hline Gap-box & Arabidopsis thaliana & 1365 & CAAATGAA(A/G)A & part of a light responsive element \\
\hline HSE & Brassica oleracea & 1224 & AGAAAATTCG & cis-acting element involved in heat stress responsiveness \\
\hline HSE & Brassica oleracea & 1485 & AAAAAATTTC & cis-acting element involved in heat stress responsiveness \\
\hline LAMP-element & Pisum sativum & 588 & CTTTATCA & part of a light responsive element \\
\hline TATA-box & Arabidopsis thaliana & 1408 & ccTATAAAaа & core promoter element around -30 of transcription start \\
\hline TATA-box & Arabidopsis thaliana & 1410 & TATAAA & core promoter element around -30 of transcription start \\
\hline TATA-box & Lycopersicon esculentum & 1412 & TTTTA & core promoter element around -30 of transcription start \\
\hline TATA-box & Lycopersicon esculentum & 1496 & TTTTA & core promoter element around -30 of transcription start \\
\hline TC-rich repeats & Nicotiana tabacum & 731 & АТTТTCTTCA & cis-acting element involved in defense and stress responsiveness \\
\hline TC-rich repeats & Nicotiana tabacum & 810 & ATTTTCTTCA & cis-acting element involved in defense and stress responsiveness \\
\hline TCA-element & Nicotiana tabacum & 1256 & ССАТСТTTTT & cis-acting element involved in salicylic acid responsiveness \\
\hline TGA-element & Brassica oleracea & 1062 & AACGAC & auxin-responsive element \\
\hline circadian & Lycopersicon esculentum & 9 & CAANNNNATC & cis-acting regulatory element involved in circadian control \\
\hline circadian & Lycopersicon esculentum & 1208 & CAAAGATATC & cis-acting regulatory element involved in circadian control \\
\hline circadian & Lycopersicon esculentum & 1108 & CAANNNNATC & cis-acting regulatory element involved in circadian control \\
\hline
\end{tabular}

Table 2. Transcription factor binding sites in PIP1 aquaporin promoter

\begin{tabular}{|c|c|c|c|c|c|}
\hline Factor & Site & Strand & Seq & Species & Source \\
\hline AGL 3 & 443 & - & atcaCCATAcaatcacca & Arabidopsis thaliana & TRANSFAC \\
\hline AGL 3 & 505 & - & attaCCATAtacacaaca & Arabidopsis thaliana & TRANSFAC \\
\hline AGL 3 & 1229 & - & tagttaagTATGGaatt & Arabidopsis thaliana & TRANSFAC \\
\hline AGL 3 & 1412 & - & tgttgttttTATGGtaac & Arabidopsis thaliana & TRANSFAC \\
\hline $\mathrm{AG}$ & 85 & - & tttaaatatTTTGGtttg & Arabidopsis thaliana & TRANSFAC \\
\hline $\mathrm{AG}$ & 573 & - & aacaCCAAAattgtgtgc & Arabidopsis thaliana & TRANSFAC \\
\hline $\mathrm{AG}$ & 610 & - & tgcaCCAAAgagacaaat & Arabidopsis thaliana & TRANSFAC \\
\hline $\mathrm{AG}$ & 917 & - & tggaCCAAAgagacaaa & Arabidopsis thaliana & TRANSFAC \\
\hline $\mathrm{AG}$ & 1107 & - & taaactaaaTTTGGcaaa & Arabidopsis thaliana & TRANSFAC \\
\hline $\mathrm{AG}$ & 1188 & - & ttttCCAAAtacacattc & Arabidopsis thaliana & TRANSFAC \\
\hline $\mathrm{AG}$ & 1567 & - & gattCCAAAattgtcaat & Arabidopsis thaliana & TRANSFAC \\
\hline $\mathrm{AG}$ & 1729 & - & gttattttcTTTGGcata & Arabidopsis thaliana & TRANSFAC \\
\hline Athb-1 & 102 & - & tgtcaATTATtgtg & Arabidopsis thaliana & TRANSFAC \\
\hline Athb-1 & 206 & - & cttaaATTAttga & Arabidopsis thaliana & TRANSFAC \\
\hline Athb-1 & 890 & - & aaaaATAATttaa & Arabidopsis thaliana & TRANSFAC \\
\hline
\end{tabular}


(pGRAqup) were confirmed via restriction (Fig. 2b) and PCR (Fig. 2c). Clones were also confirmed by DNA sequencing on an ABI3100 DNA sequencer using BigDye Terminator sequencing kit version 3.1. The final plasmid map of pGRAqup is shown in Fig. 3.

Cloning of PIP1 gene promoter in plant transformation vector pGA482. The vector pGA482 was ligated successfully to the complete expression cassette of aquaporin PIP1 promoter-GuS-CaMV terminator that was obtained from pGRAAqup. The resultant pGA482 construct were named as pGAAqup. The construct confirmation was done by restricting the pGAAqup with Hind III (Fig. 4).

Agrobacterium-mediated tobacco transformation. pGAqup was transformed into the Agrobacterium (LBA4404) elcetrocompetent cells by electroporation. The clones were confirmed by PCR using reverse and forward promoter specific primers. Leaf discs of Nicotiana tabaccum were cut and co-cultivated with the cultured Agrobacteroium containing plant expression vector pGAAqup. This gives a fair chance to the Agrobacterium to transform the gene of interest into the plant. Leaf disc were placed on solidified MS0 medium till shooting and rooting (Fig. 5).

Transgenic analysis. Multiple transgenic plants were obtained through Agrobacterium-mediated transformation. However, a few plants were selected randomly

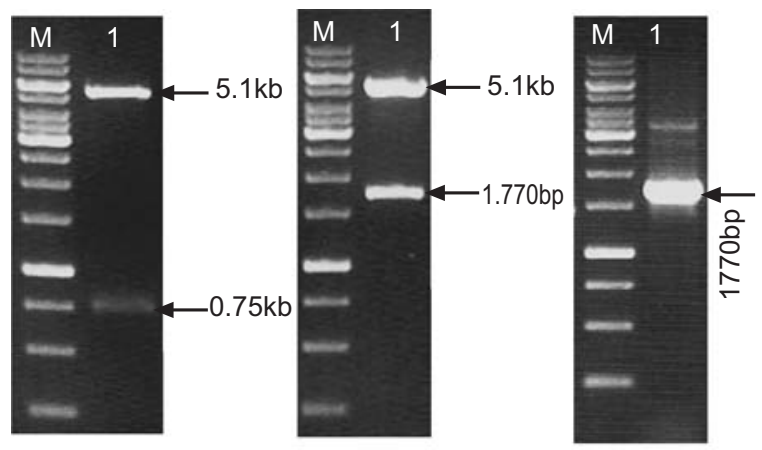

Fig. 2. Confirmation of pGR1 vector and pGR Aqup. (a) M: $1 \mathrm{~Kb}$ DNA ladder, lane 1: digestion of pGRI1 vector with SacI and Hind III released about $0.75 \mathrm{~kb}$ fragment; (b) M: $1 \mathrm{~Kb}$ DNA ladder, lane 1: digestion of pGRAqup clone with SacI and Hind III released 1770bp fragment and (c) M: $1 \mathrm{~Kb}$ DNA ladder, lane 1: PCR amplification of aquaporin promoter. for transgene analysis. The total genomic DNA was isolated from the putative transgenic and nontransformed negative control plants using CTAB method. The transgenics were confirmed by PCR using promoter specific primers. Results of PCR analysis are shown in Fig. 6. The results show amplification of expected fragments from the transgenics for PIP1 construct. The amplifications in the transgenics yielded identical size of amplified DNA fragment that could also be seen in the positive control. However, no amplification was observed in the genomic DNA of control plant.

Evaluation of PIP1 promoter by GUS assay. The specificity and efficiency of the PIP1 promoter was evaluated through transient GUS assay in different tobacco plant tissues. The plasmid from clone pGRAquP was used to coat gold particles. The particle bombard-

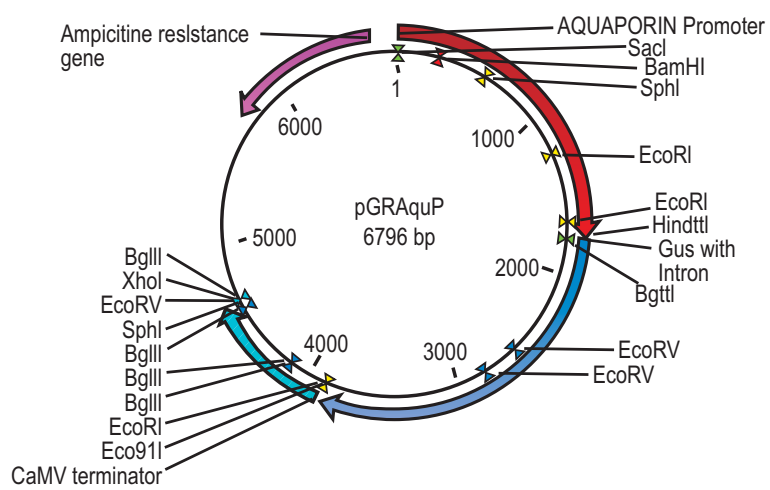

Fig. 3. pGRAqup: vector map of aquaporin promoter controlling GUS expression in pGR1.

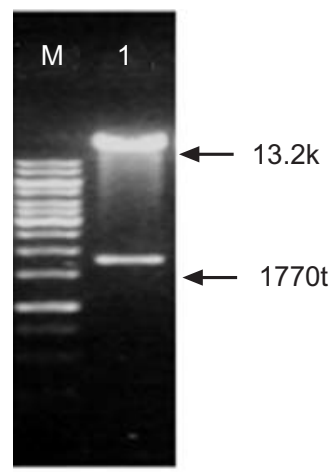

Fig. 4. (a) Lane M: $1 \mathrm{~Kb}$ DNA ladder, lane 1: confirmation of aquaporin expression cassette in pGA482 by digestion with SacI and Xhol(b) lane M: $1 \mathrm{~kb}$ DNA ladder. 

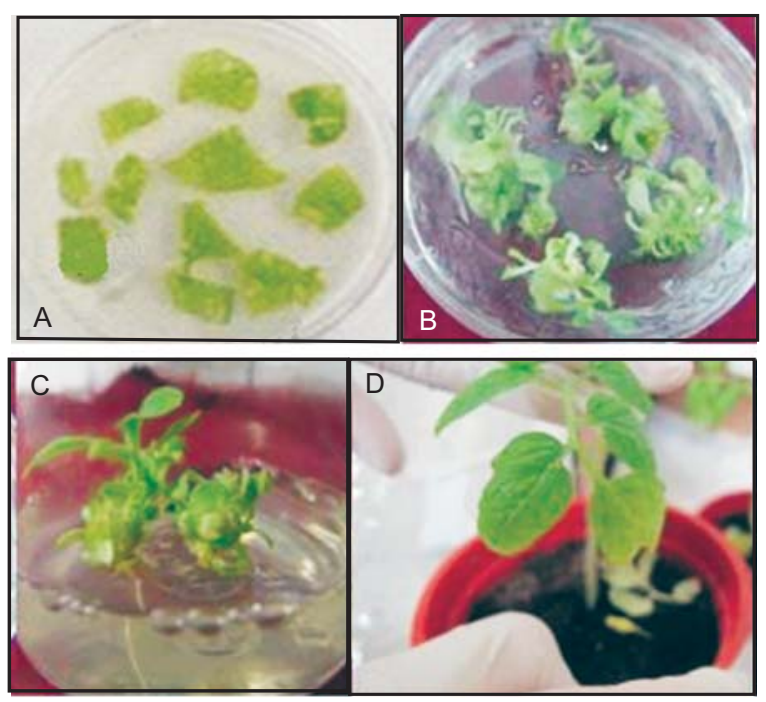

Fig. 5. Different stages of Agrobacterium-mediated tobacco transformation (a) tobacco leaf discs for co-cultivation with Agrobacterium inoculums; (b) tiny plantlets appearing after 7-10 days of tobacco leaf discs co-cultivation with Agrobacterium inoculum; (c) excised 7 days old plantlets which survived on Kanamycin selection media shifted to jar and (d) mature plantlets shifted to soil.

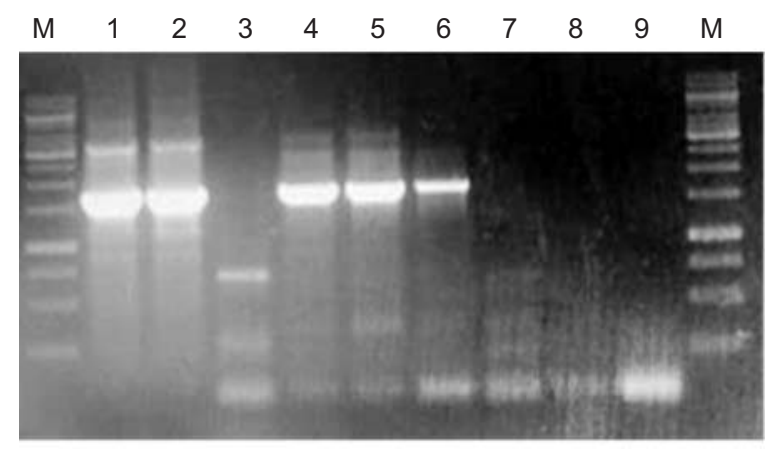

Fig. 6. PCR analysis of putative transgenic tobacco plants for PIP1 promoter using promoter specific primers. M: $1 \mathrm{~kb}$ DNA ladder, Lane 1: PCR of positive control using plasmid DNA of pGRAqup as a template, Lane 2: PCR of positive control using plasmid DNA of pGAAqup as a template, Lane 3: Negative control of tobacco, Lane 4-7: PCR analysis of putative transgenic plants using promoter specific primers, showing expected amplification product of $1770 \mathrm{bp}$, Lane 8: Negative control of tobacco and Lane 9: Negative control of PCR master mis and water. ment experiments were conducted on leaves and stem tissue of tobacco along with the positive and negative controls. The bombarded tissues were observed under the microscope after appropriate incubation in the GUS staining solution. The stained tissues were photographed with a Polaroid digital camera. The microscopic studies revealed that the PIP1 promoter constitutively expressed in all the selected tissues (Fig. 7).

The leaf tissues from PCR selected stable transgenic plants for PIP1 promoter were stained for GUS activity. The staining reaction showed that PIP1 promoter was constitutively expressed in leaves. Longer incubation in the staining solution led to diffusion of the stain but did not reveal any increase of GUS activity or spreading
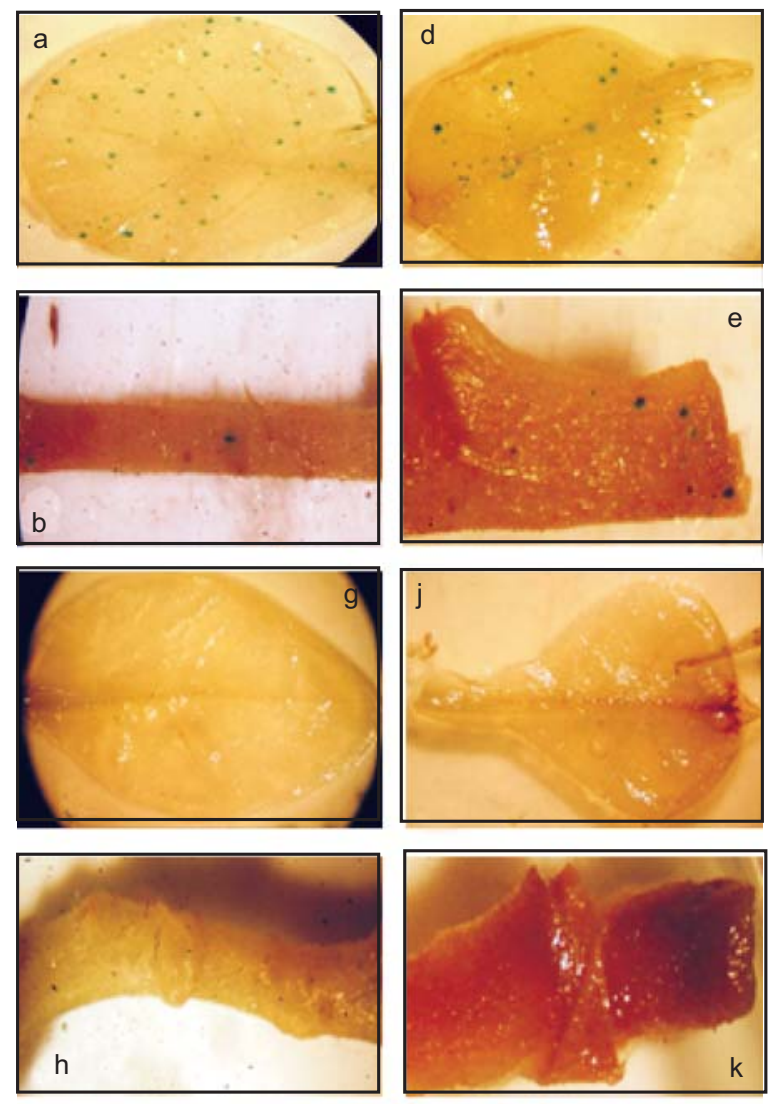

Fig. 7. Transient GUS expression analysis of aquaporin promoter in tobacco tissues. (a-b) GUS activity under aquaporin promoter in tobacco leaf and stem, (d-e) positive control: GUS activity under $2 \times 35 \mathrm{~S}$ promoter in tobacco leaf and stem, (g-h) Negative control: P tobacco leaf and stem bombarded with uncoated gold particles, (j-k) Negative control: Un-bombarded but GUS stained tobacco leaf and stem. 
of stain in the tissues. The GUS staining indicated that the promoter PIP1 shows comparable expression in different transgenic events (Fig. 8 and 9). The staining of leaves from non-transgenic plants did not reveal the development of GUS stain.

Identification of new useful promoters is required to generate resources for an optimized and tissue specific expression of the genes of interest for improving crop traits. Some already reported and most commonly used gene promoters that have constitutive type expression include CaMV 35S and the maize and soybean polyubiquitin promoters (Chiera et al., 2007; Jani et al., 2002; Christensen et al., 1992). However, to expand the availability of promoters for driving effective constitutive gene expression more promoter sequences are required to be explored for utilization in transgene development programs.

Aquaporins are involved in different developmental and regulatory mechanisms of plants including water permeability, cell elongation, fertilization, stomatal

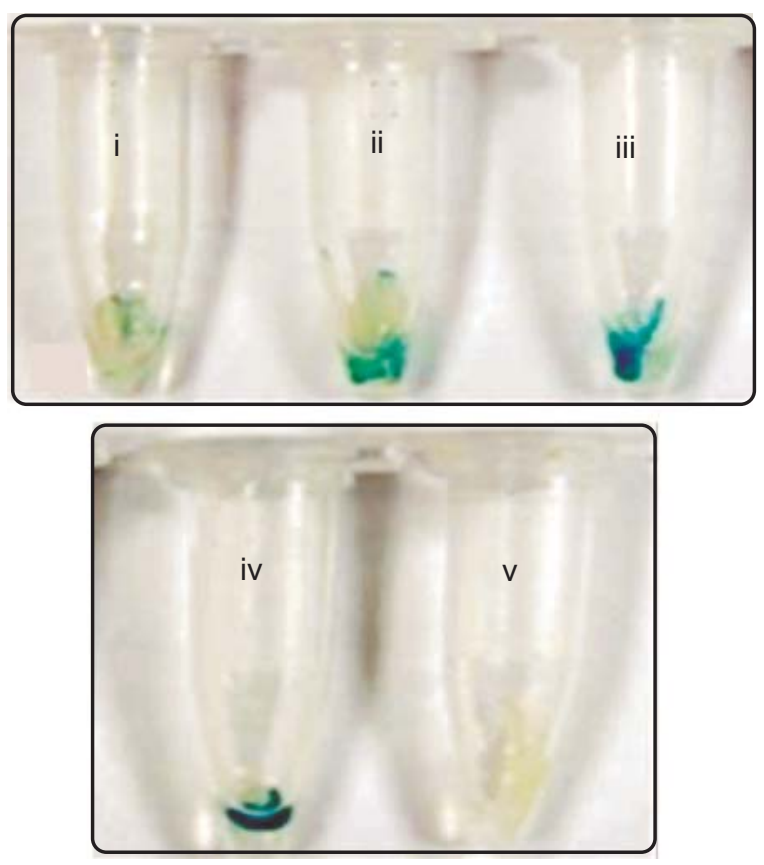

Fig. 8. Histochemical assay for GUS activity in stably transformed tobacco plants. Tubes (i-iii) Leaf tissue stained from different transgenic events for GUS activity controlled by aquaporin promoter: tube (iv) GUS activity in tobacco leaves controlled by $2 \times 35 \mathrm{~S}$ : (v) shows non trangenic i.e. negative control. opening, and seed germination (Maurel et al., 2009). Therefore, the present study was designed to identify and characterize a highly expressed plant gene PIP1 aquaporin promoter from dicot plant, S. tubersosum.

Many cis-regulatory motifs were detected in the PIP1 promoter sequence along with enhancer regions (CAATbox) and core promoter element transcription start site (TATA-box). Gap-box, ATCT motif, Box 4, LAMPelement, AE-box, and GA-motif are the light responsive elements which have been found in many light inducible gene promoters of Arabidopsis (Conley et al., 1994). The presence of these light responsive elements in PIP1 promoter suggested that this promoter might be involved in light induction in photosynthetic tissues. Phytohormone, stress and defense regulatory elements found in PIP1 promoter include auxin responsive element (AuxRR-core), TGA-element, TCA element, TC rich repeats and heat responsive element (Bernard et al., 2010; Nemhauser et al., 2004; Rizhsky et al., 2004). The regulatory region of PIP1 aquaporin promoter containing such phytohormone, stress and defense regulatory elements indicates its role in stress regulated gene expression. The analyses of most of the motifs
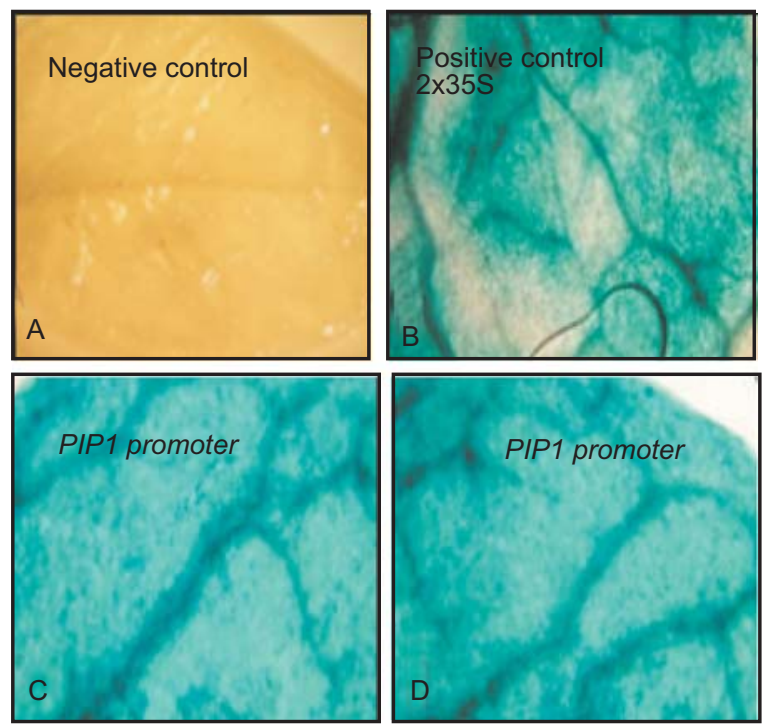

Fig. 9. Close view of histochemical assay for GUS activity in stably transformed tobacco plants. (a) Non transgenic i.e. negative control, (b) GUS activity in leaves controlled by $2 \times 35 \mathrm{~S}$, (c-d) Close view of leaf tissue stained from different transgenic events for GUS activity controlled byu PIP1 aquaporin promoter. 
associated with the PIP1 aquaporin promoter indicate that this promoter can express genes in all the tissues in plants and is regulated by light as well as stress response. In other words, it can be inferred that this promoter is rather having a constitutive response. This inference is supported by the fact that GUS expression is detected in all the selected tissues. The transient studies revealed that PIP1 promoter has shown GUS expression in all the leaf and stem of tobacco. Whereas, the stable tobacco lines for PIP1 promoter showed a strong expression of GUS in tobacco leaves.

\section{Conclusions}

It is concluded that PIP1 promoter can be utilized to confer a constitutive gene expression in plants. The $2 \times 35 \mathrm{~S}$ promoter has been reported to exhibit strong constitutive activity in different plant species and the same was observed in our experiments. The relative comparison of the PIP1 promoter with $2 \times 35$ S promoter indicated that both were constitutively expressed in different tobacco tissues. It is proposed that the promoter identified through this study may be utilized to overcome the gene silencing problem by reducing the foreign gene expression to a lower level than the $2 \times 35 \mathrm{~S}$.

\section{Acknowledgement}

We are thankful to Dr. Shahid Mansoor, SI Director NIBGE, for full support and our group members for all the valuable suggestions. We are thankful to all authors for their kind support. All authors read and approved the final manuscript.

Conflict of Interest. The authors declare no conflict of interest.

\section{References}

Battraw, M.J., Hall, T.C. 1990. Histochemical analysis of CaMV $35 \mathrm{~S}$ promoter- 3 -glucuronidase gene expression in transgenic rice plants. Plant Molecular Biology, 15: 527-538.

Bernard, V., Brunaud, V., Lecharny, A. 2010. TC-motifs at the TATA-box expected position in plant genes: a novel class of motifs involved in the transcription regulation. BMC Genomics, 11: 166.

Bots, M., Vergeldt, F., Wolters-Arts, M., Weterings, K., van As, H., Mariani, C. 2005. Aquaporins of the PIP2 class are required for efficient anther dehiscence in tobacco. Plant Physiology, 137: 10491056.
Chaumont, F., Barrieu, F., Wojcik, E., Chrispeels, M. J., Jung, R. 2001. Aquaporins constitute a large and highly divergent protein family in maize. Plant Physiology, 125: 1206-1215.

Chiera, J.M., Bouchard, R.A., Dorsey, S.L., Park, E., Buenrostro-Nava, M.T., Ling, P.P., Finer, J.J. 2007. Isolation of two highly active soybean Glycine max (L.) Merr. promoters and their characterization using a new automated image collection and analysis system. Plant Cell Reports, 26: 1501-1509.

Christensen, A.H., Quail, P.H. 1992. Sequence analysis and transcriptional regulation by heat shock of polyubiquitin transcripts from maize. Plant Molecular Biology, 12: 619-632.

Conley, T.R., Park, S.C., Kwon, H.B., Peng, H.P., Shih, M.C. 1994. Characterization of cis-acting elements in light regulation of the nuclear gene encoding the a subunit of chloroplast isozymes of glyceraldehyde-3-phosphate dehydrogenase from Arabidopsis thaliana. Molecular and Cellular Biology, 14: 2525-2533.

Jani, D., Meena, L.S., Rizwan-ul-Haq, Q.M., Singh, Y., Sharma, A.K., Tyagi, A.K. 2002. Expression of cholera toxin B subunit in transgenic tomato plants. Transgenic Research, 11: 447-454.

Johanson, U., Gustavsson, S. 2002. A new subfamily of major intrinsic proteins in plants. Molecular Biology and Evolution, 19: 456-461.

Johanson, U., Karlsson, M., Johansson, I., Gustavsson, S., Sjövall, S., Fraysse, L., Kjellbom, P. 2001. The complete set of genes encoding major intrinsic proteins in Arabidopsis thaliana provides a framework for a new nomenclature for major intrinsic proteins in plants. Plant Physiology, 126: 1358-1369.

Lopez, F., Bousser, A., Sissoëff, I., Gaspar, M., Lachaise, B., Hoarau, J., Mahé, A. 2003. Diurnal regulation of water transport and aquaporin gene expression in maize roots: contribution of PIP2 proteins. Plant and Cell Physiology, 44: 1384-1395.

Martre, P., Morillon, R., Barrieu, F., North, G.B., Nobel, P.S., Chrispeels, M.J. 2002. Plasma membrane aquaporins play a significant role during recovery from water deficit. Plant Physiology, 130: 2101 2110 .

Maurel, C., Santoni, V., Luu, D.T., Wudick, M.M., Verdoucq, L. 2009. The cellular dynamics of plant aquaporin expression and functions. Current Opinion in Plant Biology, 12: 690-698.

Murashige, T., Skoog, F. 1962. A revised medium for 
rapid growth and bio assays with tobacco tissue cultures. Physiologia Plantarum, 15: 473-497.

Naqvi, R.Z., Mubeen, H., Raza, S. 2016. Role of plant promoters and their cis regulatory elements in gene expression regulation. European Journal of Pharmaceutical and Medical Research, 3: 347-352.

Nemhauser, J.L., Mockler, T.C., Chory, J. 2004. Interdependency of brassinosteroid and auxin signaling in Arabidopsis. PLoS Biology, 2: e258.

Quigley, F., Rosenberg, J.M., Shachar-Hill, Y., Bohnert, H.J. 2001. From genome to function: the Arabidopsis aquaporins. Genome Biology, 3: 1-1.

Rizhsky, L., Davletova, S., Liang, H., Mittler, R. 2004.
The zinc finger protein Zat12 is required for cytosolic ascorbate peroxidase 1 expression during oxidative stress in Arabidopsis. Journal of Biological Chemistry, 279: 11736-11743.

Rogers, S.O., Bendich, A.J. 1985. Extraction of DNA from milligram amounts of fresh, herbarium and mummified plant tissues. Plant Molecular Biology, 5: 69-76.

Sakurai, J., Ishikawa, F., Yamaguchi, T., Uemura, M., Maeshima, M. 2005. Identification of 33 rice aquaporin genes and analysis of their expression and function. Plant and Cell Physiology, 46: 15681577. 